\title{
Von ethnographischen Besonderheiten in St. Gallen
}

Hans Krucker

\section{Ardhanari}

Demonstration und Veranschaulichung göttlicher Allmacht und Vielgestalt sind in den verschiedenen Religionsgemeinschaften verschiedene Wege gegangen. Immer wurde dafür aber ein Ausdruck gesucht und gefunden. In den Religionen der Antike wie im Hinduismus, im nördlichen Buddhismus und im Lamaismus bestand und besteht die Vielgötterei, die sich auch auf die Trennung von männlichen und weiblichen Energien erstreckt. Auch in den christlichen Kirchen schimmert solche Differenzierung unterschiedlicher göttlicher Kräfte immer noch durch. Der eine und andere Heilige wird für dieses und jenes besonders angesprochen. Einzelne wundertätige Orte gelten als mit speziellen religiösen Heilswirkungen ausgerüstet.

Wo im Glauben an viele Götter Verwandtschaften und innere Zusammenhänge, auch Übergänge vom einen ins andere, angenommen sind, hat es nicht daran gefehlt, daß die Vorstellung von Doppelgottheiten und von Trinitäten aufgekommen ist, wobei sich solcher Synkretismus gelegentlich nicht bloß in einem Miteinander und Nebeneinander himmlischer Gestalten, sondern in einem eigentlichen Ineinander von Göttern äußert. Das Museum Guimet in Paris wartet dem Besucher mit einem Standbild Hari-Hara auf, das zum Beispiel Vishnu zusammen mit Shiva und deren Emblemen je hälftig in einer Gestalt verkörpert.

In der sanktgallischen Sammlung für Völkerkunde besteht eine andere Plastik dieser Art. Es geht um den ebenfalls hinduistischen Doppelgott Ardhanari [Bronze 17. Jahrhundert]. Ardhanari umfaßt in ein und derselben Figur sowohl Shiva, wie auch dessen, dabei in ihrer milden und gütigen Form erscheinende, Gemahlin Uma oder Parvati. Die Legende will es, daß Shiva mit ihr eine so enge körperliche Verbindung eingegangen sei, daß sein Leib mit dem ihren zusammenwuchs und daß beide auf diese Weise mit all ihren getrennten Eigenschaften in ein zweigeschlechtiges göttliches Wesen verschmolzen. Die sehr belebt wirkende Figur ist $19 \mathrm{~cm}$ hoch und hat zwischen ausgebreiteten Armen eine Breite von $22 \mathrm{~cm}$. Die Gottheit ist vierarmig, sitzend, ein Bein erscheint hochgestellt. Vom Beschauer aus gesehen gilt die linke Hälfte Shiva, die rechte seiner Ge-

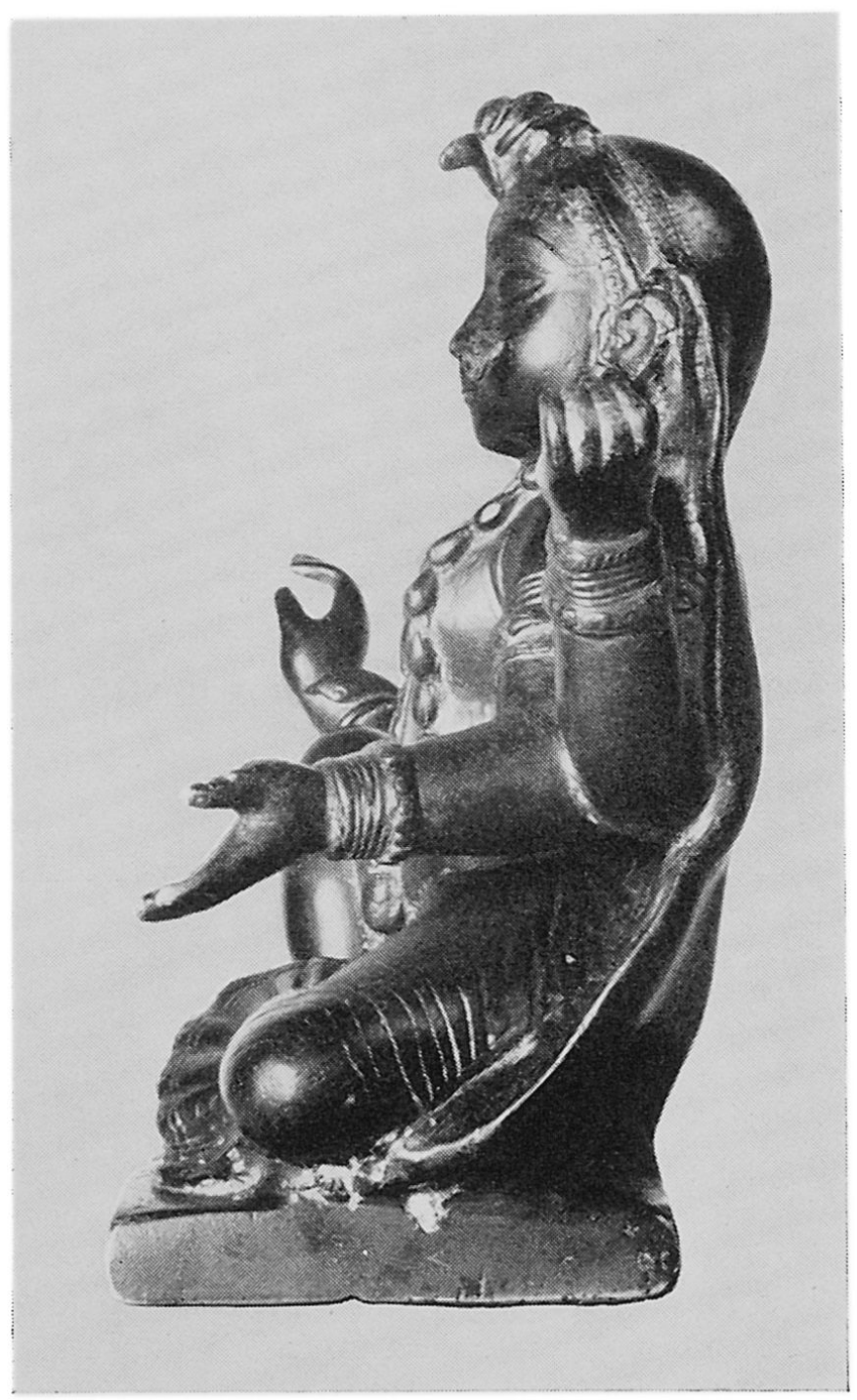

Ardhanari, indische Doppelgottheit [frauliche Seite]

mahlin. Links zeigt sich der Haarschopf des Asketen, umwunden von einer Schlange. Aus dem Haar fällt die Ganga als breiter Strom auf die Schulter herunter. Die Stirne zeigt das Sektenzeichen mit drei Strichen. Als männliches Kennzeichen ist der Schnurrbart vorhanden. Um den Hals hängt die Schädelkette, und über den Rücken fällt das Tigerfell, auf dem Shiva sitzt. In den Händen trägt er den Dreizack, schlangenumwunden, gleich wie seine Handgelenke. Der jünglingshaft wohlgebildete Körper ist unbekleidet. 


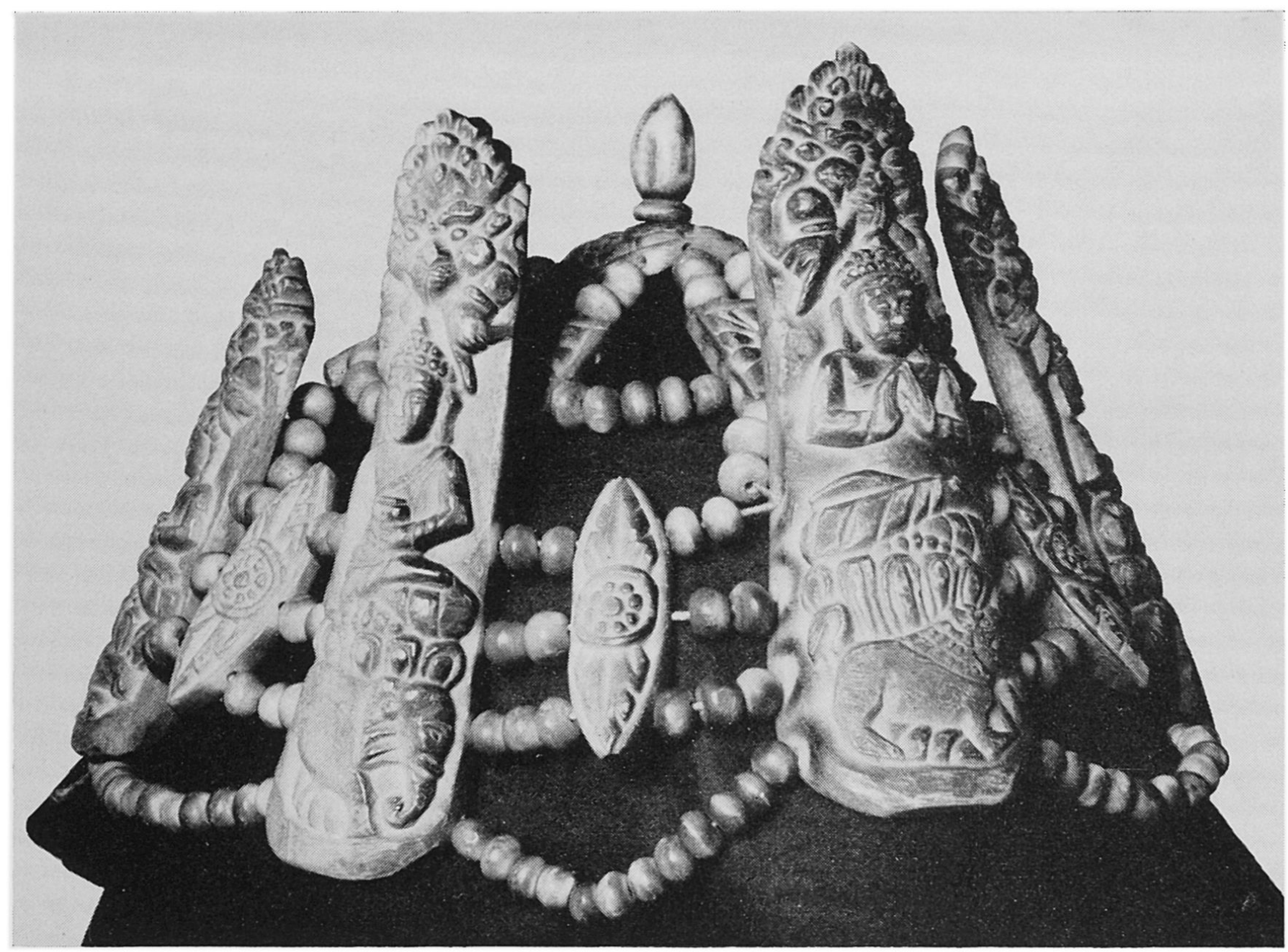

Knöcherne Priestermütze aus Tibet

Uma auf der rechten Figurenseite trägt die schlichte Haartracht; sie besitzt reichen Stirn-, Nasen- und Ohrschmuck. Ihr Gesicht ist im Gegensatz zu dem Shivas fraulich schön gerundet. Die Verschiedenheiten umfassen derart alle Details. Die Frau trägt den großen Juwelen-Halsschmuck. Arme und Beine sind mit Ringen geschmückt. Der Oberkörper ist entblößt. Die Brust tritt hervor. Von der Hüfte an trägt die Göttin ein bis zu den Füßen reichendes, faltig wallendes Gewand.

Trotz Doppelbedeutung und Doppelaussehen bietet die Bronze dem Auge ein merkwürdig in sich geschlossenes Bild dar, das sie nicht nur wissenschaftlich interessant macht, sondern das sie auch als ein wohlüberlegtes und schön empfundenes Kunstwerk erscheinen läßt.

\section{Tibetische Priestermütze}

Verehrung und Gebrauch von Reliquien entspringen der Vorstellung, daß sich mit den die Zeit überdauernden Gebeinen auch die geheimen sittli- chen Kräfte der Verstorbenen erhalten und daß diese Kräfte, empfunden als Gottesnähe, als Frömmigkeit, Wundertätigkeit, empfunden auch als Mut und Tapferkeit, weiterhin von ihnen ausstrahlen und so wirkam bleiben. Während Reliquien im katholischen Kultus nur in Heiligenschreinen der Verehrung der Gläubigen ausgesetzt sind, geht in dem, frühchristlicher Kultur ebenfalls nicht ganz verschlossen gewesenen Lamaismus Tibets alles viel weiter. Die Gebeine großer Religionsführer werden nicht nur verehrt und angebetet, vielmehr werden sie als eigentliche Gebrauchsgegenstände praktisch in den religiösen Dienst miteinbezogen. Ethnographische Sammlungen bestätigen das Gesagte auf Schritt und Tritt. So verfügen schon die nicht großen Sammlungen St. Gallens über einen kunstvoll gefaßten lamaistischen Schädelbecher, ferner über eine von Menschenhaut überspannte, von zwei Schädelkapseln gebildete Schädeltrommel, eine Tempeltrompete aus Oberschenkelknochen, eine beinerne Gebetskette, zwei beingeschnitzte kultische Armbänder, einen der bekannten tantrischen Schurze, vierreihig mit oberer Spannweite von 85 
$\mathrm{cm}$ bei 26 Knochenplaketten, welche göttliche Gestalten mit Tiersymbolen, Masken und Zaubergravierungen aufweisen: alles längs und quer durch Ketten von Beinkugeln miteinander verbunden.

Dazu geselit sich eine in europäischen Museen jedenfalls nur selten anzutreffende knöcherne Priestermütze, wie sie bei den, furchtbaren Schutzgöttern, drag-gsed, geltenden Opferriten getragen werden. Die ebenfalls netzartige, nicht mit Stoffen eingelegte Knochenmütze besitzt, entsprechend der fünfteiligen Krone der tibetischen Bodhisattvas, fünf aufstehende, leicht zugespitzte Lappen, die unter sich und über den Kopf wieder mit Kugelketten - eingeschaltet sind magische und ein ornamentales Zierplättchen — verbunden sind. Ein oben aufsitzender, alles zusammenhaltender knospenartiger Beinknauf erinnert an den Ushnisha oder die Kopfflamme Buddhas als Symbol der Erleuchtung. Die 10 bis $13 \mathrm{~cm}$ hohen, aufstehenden, die eigentliche Krone [tibetisch: cod pan] bildenden Beinlappen sind reich, und, wie es ihrem kirchlichen Zwecke zukommt, sehr, sorgfältig geschnitzt. Auf den leicht gewölbten Knochentäfelchen finden sich Darstellungen der fünf Dhyanibuddhas, sitzend auf dem Lotosthron in klassischer Haltung mit nach oben gekehrten Fußsohlen, angetan mit dem einfachen Mönchsgewand, Hände in Gebetshaltung bei der mittleren Platte, mit Almosengefäß und mit Gesten lehrend und die Erde als Zeuge anrufend, bei den seitlichen Bildnissen. Eine der Buddhagestalten ist vom vishnuitischen Schlangenbaldachin überdacht. Die Basis der Darstellungen wird von den Buddha heiligen Tieren, Elefant, Pfau, Pferd, Löwe und Schwan [?] gebildet. Über den Buddhafiguren schweben die dämonenhaften schrecklichen Schutzgötter, die drag-gsed, denen eben der Kult mit diesen Beinkappen gewidmet ist. Sie erscheinen in der zornigen, ins Fürchterliche gesteigerten Form mit dicken Gesichtern, struppigem Haar, heraustretenden Augen [auch Stirnaugen], leckender Zunge, fletschenden Zähnen und mit Klauenhänden. Die unteren Körperpartien fehlen oder sind hinter den Buddhafiguren versteckt. Jedes der Kronenblätter stellt mit seiner flachen Reliefschnitzerei ein kleines Kunstwerk für sich dar. Die Priestermütze wurde in Nepal erworben.

\section{Eine interessante Formübertragung}

Die Abbildung zeigt einen mit sehr flachem, doch deutlich konturiertem Relief in hartes $\mathrm{Holz}$ geschnittenen vierhändigen klauenbewehrten Dämon. Das Stück ist Zierplatte zu einem Krisständer aus Bali [Indonesien]. Die mit dem Rüssel und den Stoßzähnen des Elefanten versehene Gestalt trägt gleichzeitig ein um den Hals gelegtes Schlangenband. Damit verrät sich die Figur wohl als ein dem

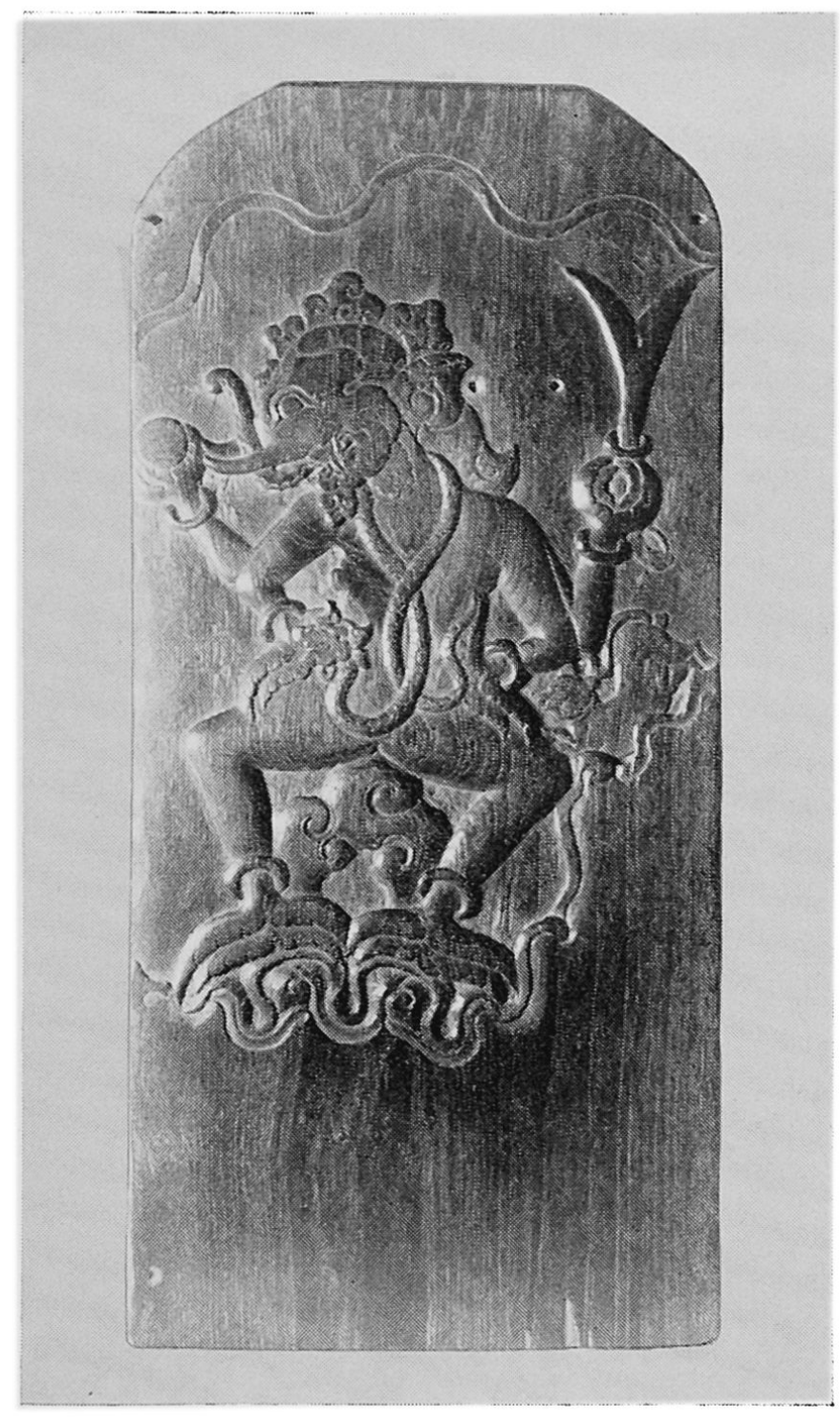

Rüsseldämon auf Zierplatte zu balinesischem Krisständer.

Hinduismus nahestehendes Bild des elefantenköpfigen Ganesha.

Im großen ethnographischen Nachbarfeld Neuguineas [unterer Sepik und Ramugebiet] besteht der eigenartige sogenannte "Schnabelstil» als künstlerisches und religiöses Formelement. Was damit gemeint ist, sind die an vielen hölzernen Ahnenfiguren, an ihnen ähnlichen, aber kleineren Amulettschnitzereien, auch an Masken anzutreffenden, bis zum Kinn oder bis zum Nabel herabreichenden, unten eingerollten "Nasen", für deren Form die bloße Annahme eines Vogelschnabels oder eines verlängerten Nasenschmuckes ungenügend wirkt. Daher ist die andere Auffassung aufgekommen, daß dieses schwungvolle verlängerte Nasengebilde der Sepikplastiken ebenfalls etwas mit dem Elefantenrüssel Ganeshas und diese Figuren mit Ganesha selbst etwas zu tun haben könnten. $\mathrm{Zu}$ solcher Úberlegung wird man auch durch die stilkritisch 
interessante Tatsache hingeführt, daß die auffallend schräggestellten Augen und Gesichtszüge sowohl an den Schnitzwerken Neuguineas wie auch an den Ganesha-Bildnissen Indiens zu sehen sind.

Der hier wiedergegebene Rüsseldämon aus Bali erscheint in solchem Zusammenhang geographisch und inhaltlich als ein interessantes Zwischen- und Bindeglied. Einmal ist es von ihm weg nur ein kleiner Schritt zu dem ja auch in Java beliebt gewesenen göttlichen Ganesha. Sodann liegt es nahe, daß, wenn das Ganesha-Rüsselmotiv in die Dämonenwelt Balis hat Eingang finden können, es ebensogut möglich ist, daß dies auch für die Kultfiguren Neuguineas Gültigkeit hat. Die Krisplatte darf als Beleg dafür angesehen werden, daß solche Wirkungen in die Südsee hinaus vorerst wohl in Indonesien fußten und daß die dem «Schnabelstil» Neuguineas gegebene neue Auslegung ihre Richtigkeit hat.

\section{Résumé}

Les œuvres représentées se rapportent d'abord à une figure en bronze hindouiste Ardhanari, du $17 \mathrm{e}$ siècle, qui, en tant que double représentation di- vine, montre à d'une part Shiva à la coiffure d'Ascètes et, d'autre part, son épouse sous les traits bienveillants de Uma ou Parvati. La réproduction d'une divinité bi-sexuelle munie de tous ses emblèmes est une découverte ethnologique très rare. $\mathrm{Ce}$ bronze donne l'impression d'une œuvre très caractéristique et complète.

On décrit, en second lieu, un chapeau de prêtre en os, pièce également peu courante, provenant de l'inventaire religieux d'un temple des Lamahs; cette pièce porte 5 tablettes d'os verticales sculptées, représentant la couronne des Bodhisattvas tibétiens. Des chapeaux en os de cette espèce servaient au culte consacré aux Drag-gsed, terribles dieux-protecteurs de la foi lamaïste.

Le Ganesha à tête d'éléphant joue un rôle important dans l'art de l'Inde et de l'Indonésie. On est tenté de croire que la représentation si curieuse du nez allant du menton au nombril, que l'on rencontre dans les statues d'ancêtres et dans les masques de la région des fleuves Sepik et Ramu de la Nouvelle-Guinée, a quelque chose de commun avec le Ganesha. L'auteur voit une confirmation de cette opinion dans un plat sculpté balinésien d'un support Kris qui représente le portrait d'un démon avec une trompe, des défenses et une chaîne de serpents. On peut tout à fait s'imaginer que le motif de la trompe soit passé de là dans la mythologie et dans la conception artistique de la Nouvelle-Guinée. 